\title{
A LOCAL SPECTRAL THEORY FOR OPERATORS. III: RESOLVENTS, SPECTRAL SETS AND SIMILARITY
}

\author{
BY \\ J. G. STAMPFLI $\left({ }^{1}\right)$
}

\begin{abstract}
Let $T$ be a bounded linear operator on a Hilbert space and assume $T$ has thin spectrum. When is $T$ similar to a normal operator? This problem is studied in a variety of situations and sufficient conditions are given in terms of characteristic functions, resolvents, spectral sets, and spectral resolutions. By contrast, the question "When is $T$ normal?" has a relatively simple answer since in that case a necessary and sufficient condition can be given in terms of the resolvent alone.
\end{abstract}

If an operator $T$ on a Banach space has its spectrum on a smooth curve, then a very weak growth condition on the resolvent $(\lambda-T)^{-1}$ is sufficient to ensure a plenitude of invariant subspaces (see [1], [12], [15], [16], [17], [25], [40], and [41]). There has been considerable interest recently in finding conditions on the operator which would guarantee a spectral theory of the Dunford type. The greatest success has been achieved when the underlying space is a Hilbert space. We now quote three results which lend perspective to the subsequent material.

From here on, all operators are bounded transformations on a Hilbert space $\mathscr{H}$. We designate $(\lambda-T)^{-1}$ by $R_{\lambda}(T)$.

THEOREM 0 [29]. Let the spectrum of $T$ lie in a $C^{2}$ Jordan curve $\Gamma$. Let $\left\|R_{\lambda}(T)\right\|$ $\leqq\{\text { dist }[\lambda, \Gamma]\}^{-1}$ for $\lambda \in M$ where $M$ is an open set containing $\Gamma$. Then $T$ is normal.

THEOREM N-F [34]. Let $T$ be a contraction. Let $\sup _{|\lambda|<1}\left\|\Theta_{T}(\lambda)^{-1}\right\|<\infty$ where $\Theta_{T}(\lambda)$ is the characteristic function of $T$. Then $T$ is similar to a unitary operator.

For a contraction $T$ the characteristic function is defined as

$$
\Theta_{T}(\lambda)=\left[-T+D_{T^{*}}\left(1-\lambda T^{*}\right)^{-1} D_{T}\right] \mid \mathscr{D}_{T}
$$

when $D_{T}=\left(1-T^{*} T\right)^{1 / 2}, D_{T^{*}}=\left(1-T T^{*}\right)^{1 / 2}$ and $\mathscr{D}_{T}=\left(D_{T^{*}} \mathscr{H}\right)^{-}$. Using Theorem N-F as a starting point, Gohberg and Kreìn obtained

THEOREM G-K [13]. Let $T$ be a contraction. Let $\left\|R_{\lambda}(T)\right\| \leqq K /(1-|\lambda|)$ for $|\lambda|<1$. Then $T$ is similar to a unitary.

In an effort to avoid the condition that $T$ is a contraction, Sahnovič [24] and Davis and Foiaș [3] have introduced a generalized characteristic function. In the

Received by the editors May 12, 1971.

AMS 1969 subject classifications. Primary 4710, 4730, 4740; Secondary 4735.

Key words and phrases. Operator, Hilbert space, resolvent, characteristic function, spectral set, similarity, spectral type operator.

(1) The author gratefully acknowledges the support of the National Science Foundation. Copyright (C) 1972, American Mathematical Society 
papers cited, they show that an operator is similar to a unitary when this new characteristic function is suitably bounded.

In this paper we attempt to extend the quoted results to a wider class of operators (and escape from the unitary operators). There are three reasonable conditions to place on an operator $T$ in an effort to obtain a spectral theory. One may ask that

(1) $\Theta_{T}(\lambda)$ be bounded,

(2) a super set of the spectrum be a spectral set,

(3) $\left\|R_{\lambda}(T)\right\| \leqq\{\text { dist }[\lambda, \sigma(T)]\}^{-1}$.

The difficulty with (1) is that it is only appropriate for operators with spectrum on the unit circle. Moreover, it is extremely difficult to compute $\Theta_{T}(\lambda)$ for a given operator. (However, the power of the characteristic function approach cannot be overstated. Theorem N-F is the basis for a good portion of this paper.) Condition (2) can be applied to any operator, but it too is difficult to check in practice. Condition (3) is weaker than (2) and much easier to verify.

The paper proceeds as follows. In $\$ 2$ and $\$ 3$ we prove Theorem G-K and a relative under slightly weaker conditions (namely (3) in place of (2)). In $\$ 6$ we extend Theorem G-K to operators with spectra on a smooth curve. Operators with very thin spectra are treated in $\$ 4$. $\S 5$ is devoted to a fairly involved example. $\$ 7$ deals with the behavior of spectral operators and answers a question raised by Nelson Dunford. A summary and some open questions can be found in $\S 8$ and $\S 9$.

Roughly speaking, the following conditions are relevant to the types of behavior we are studying: (a) the smoothness of the curve containing the spectrum, (b) the density of the spectrum itself, (c) conditions of type (1), (2) and (3) from above, and (d) additional hypotheses of a spectral nature. We attempt to make the presentation reasonably complete along those lines. Some section jumping may be necessary, however, to pursue a specific question.

I would like to express my warm thanks to Ciprian Foiaș for several stimulating discussions and for an observation which significantly shortens the original proof of Theorem 2.

1. Preliminary results. In this section we collect some known results and add a few easy propositions which will be needed later. Proofs of the known results have been included for completeness since they are so brief.

LEMMA 1 [29]. Let $\left\|(\lambda-T)^{-1}\right\| \leqq 1 / d$ where $0<d<|\lambda|$. Then

$$
\left\|\left(T^{-1}-\frac{\lambda}{|\lambda|^{2}-d^{2}}\right)^{-1}\right\| \leqq \frac{1}{d /\left(|\lambda|^{2}-d^{2}\right)} .
$$

Proof. The hypothesis is equivalent to the relation $\left(T^{*}-\bar{\lambda}\right)(T-\lambda) \geqq d^{2}$. If we expand and multiply by $T^{*-1}$ and $T^{-1}$ in the appropriate manner we find that $\left(|\lambda|^{2}-d^{2}\right) T^{*-1} T^{-1}-\bar{\lambda} T^{*-1}-\lambda T^{-1}+1 \geqq 0$. Hence

$$
\left(T^{*-1}-\frac{\lambda}{|\lambda|^{2}-d^{2}}\right)\left(T^{-1}-\frac{\lambda}{|\lambda|^{2}-d^{2}}\right) \geqq\left(\frac{d}{|\lambda|^{2}-d^{2}}\right)^{2}
$$

from which the desired conclusion is immediate. 
The last lemma is useful as a "mapping theorem" for resolvents.

The next lemma appears implicitly in [27] and explicitly in [23].

Lemma 2. Let $\lim _{n \rightarrow \infty}\left|\lambda_{n}-\lambda_{0}\right|\left\|\left(T-\lambda_{n}\right)^{-1}\right\|=1$ where $\lambda_{n} \rightarrow \lambda_{0}\left(\right.$ and $\left.\lambda_{n} \notin \sigma(T)\right)$. If $T f=\lambda_{0}$ f then $T^{*} f=\lambda_{0} f$.

Proof. It is sufficient to consider the case $\lambda_{0}=0$. Let $T^{*} f=g$. Then $\left(T^{*}-\bar{\lambda}_{n}\right) f$ $=g-\bar{\lambda}_{n} f$ and hence $\lambda_{n}\left(T^{*}-\bar{\lambda}_{n}\right)^{-1} f=f+\left(T^{*}-\bar{\lambda}_{n}\right)^{-1} g$. Since $f$ is orthogonal to $\left(T^{*}-\bar{\lambda}_{n}\right)^{-1} g$ we obtain

$$
\left\|\left(T^{*}-\bar{\lambda}_{n}\right)^{-1} g\right\|^{2}+\|f\|^{2}=\left|\bar{\lambda}_{n}\right|^{2}\left\|\left(T^{*}-\bar{\lambda}_{n}\right)^{-1} f\right\|^{2} .
$$

Hence $\lim _{n \rightarrow \infty}\left\|\left(T^{*}-\bar{\lambda}_{n}\right)^{-1} g\right\|=0$. But then $\|g\|=\left\|\left(T^{*}-\bar{\lambda}_{n}\right)\left(T^{*}-\bar{\lambda}_{n}\right)^{-1} g\right\| \rightarrow 0$, which completes the proof.

Corollary 1. Let $\sigma(T)$ lie on a $C^{1}$ curve $\Gamma$. Let $\left\|\left(\lambda_{n}-T\right)^{-1}\right\|=\left\{\operatorname{dist}\left[\lambda_{n}, \Gamma\right]\right\}^{-1}$ for $\lambda_{n} \rightarrow \lambda_{0} \in \Gamma$. If $T f=\lambda_{0} f$ then $T^{*} f=\lambda_{0} f$.

The proof of the lemma contains the following result as a special case.

Corollary 2. Let $\left\|(T-\mu)^{-1}\right\|=|\mu-\lambda|^{-1}$ for fixed $\lambda, \mu$ with $\mu \neq \lambda$. If $T f=\lambda_{0} f$ then $T^{*} f=\bar{\lambda} f$. (Moreover, $\lambda \in \operatorname{Bdry} \sigma(T)$.)

The next lemma, while extremely simple, does not seem to have a convenient reference in the literature. It reveals that the last corollary is not "special."

LemmA 3. Let $\left\|\left(T-\lambda_{1}\right)^{-1}\right\| \leqq\left|\lambda_{1}-\lambda_{0}\right|^{-1}$. Let $\lambda$ be a point on the line segment joining $\lambda_{0}$ to $\lambda_{1}$. Then $\left\|(T-\lambda)^{-1}\right\| \leqq\left|\lambda-\lambda_{0}\right|^{-1}$.

Proof. The hypothesis is equivalent to the inequality $\left\|\left(T-\lambda_{0}\right) f\right\| \geqq\left|\lambda_{1}-\lambda_{0}\right|\|f\|$ for all $f \in \mathscr{H}$. But then $\|(T-\lambda) f\| \geqq\left\|\left(T-\lambda_{1}\right) f\right\|-\left|\lambda-\lambda_{1}\right|\|f\|=\left|\lambda-\lambda_{0}\right|\|f\|$, which completes the proof.

REMARK. Lemma 3 may also be proved as follows. The hypothesis is equivalent to the statement: the set $\left\{z:\left|z-\lambda_{1}\right| \geqq\left|\lambda_{1}-\lambda_{0}\right|\right\}$ is a spectral set for $T$. The conclusion is equivalent to the fact that $\left\{z:|z-\lambda| \geqq\left|\lambda-\lambda_{0}\right|\right\}$, a super set of the above set, is a spectral set for $T$. (Spectral set is defined in $\S 6$.)

If we combine the last lemma with Theorem 0 we obtain the following amusing

Corollary. Let $\sigma(T) \subset(0,1)$. Let $\left\|R_{\lambda}(T)\right\| \leqq|\operatorname{Im} \lambda|^{-1}$ for $\lambda \in S$ where every vertical line intersecting $[0,1]$ intersects $S$ both above and below the real axis. Then $T$ is selfadjoint.

2. Operators similar to unitary operators. In this section we will show that, in Theorem G-K, the condition that the disc $D$ is a spectral set for $T$ can be replaced by a weaker condition on the growth of $R_{\lambda}(T)$. Some of the results in this section were previously announced in [30].

Definition. An operator $T$ on a Hilbert space $\mathscr{H}$ is of class $\mathscr{C}_{\rho}$ if there exists a Hilbert space $\mathscr{K} \supset \mathscr{H}$ such that $T^{n} f=\rho P_{\mathscr{H}} U^{n} f$ for every $f \in \mathscr{H}$ where $U$ is a unitary operator in $\mathscr{K}$ and $P_{\mathscr{H}}$ is the orthogonal projection of $\mathscr{K}$ on $\mathscr{H}$. 
In $\S 2$ and $\S 5$ we will have considerable need to refer to results of Sz.-Nagy and Foiaș. We shall usually refer to the original papers. However, all the material is contained in their book [37] as well.

From now on we will designate the unit disc by $D$ and the unit circle by $U$.

THEOREM 1. Let $\sigma(T) \subset D$. Let $\left\|R_{\lambda}(T)\right\| \leqq(|\lambda|-1)^{-1}$ for $1<|\lambda|<\beta$. Then $T$ is similar to a contraction.

Proof. The hypotheses imply that $T \in \mathscr{C}_{\rho}$ (see [35]). However, any $T \in \mathscr{C}_{\rho}$ is similar to a contraction by [36].

Corollary. Let $\sigma(T) \subset\{z:|z| \geqq 1\}$. Let $\left\|R_{\lambda}(T)\right\| \leqq(1-|\lambda|)^{-1}$ for $\alpha<|\lambda|<1$. Then $T$ is similar to an expansion, i.e. $T=Q S Q^{-1}$, where $\|S f\| \geqq\|f\|$ for all $f \in \mathscr{H}$.

Proof. An application of Lemma 1 permits us to apply the theorem.

THEOREM 2. Let the spectrum of $T$ be contained in $U$. Let

(a) $\left\|R_{\lambda}(T)\right\| \leqq K /(1-|\lambda|)$ for $\alpha<|\lambda|<1$, and

(b) $\left\|R_{\lambda}(T)\right\| \leqq(|\lambda|-1)^{-1}$ for $\beta<|\lambda|<1$.

Then $T$ is similar to a unitary operator.

Proof. It follows from Theorem 1 that $T=Q C Q^{-1}$ where $C$ is a contraction. Clearly, $\left\|R_{\lambda}(T)\right\| \leqq K^{\prime} /(1-|\lambda|)$ for $\alpha<|\lambda|<1$. But Theorem G-K tells us that $C$, and hence $T$, is similar to a unitary.

In view of Lemma 3 we have the following

Corollary. Let $\sigma(T) \subset U$. Let

(a) $\left\|R_{\lambda}(T)\right\| \leqq K /(1-|\lambda|)$ for $\alpha<|\lambda|<1$, and

(b) $\left\|R_{\lambda}(T)\right\| \leqq 1 /(1-|\lambda|)$ for $\lambda \in S$ when $S$ is a Jordan curve with $U$ in its interior.

Then $T$ is similar to a unitary.

REMARK. By virtue of Lemma 1 the $K$ and the 1 can be interchanged in hypotheses (a) and (b) of the theorem. Moreover, it is easy to see that conditions (a) and (b) are not needed in an annulus but merely in a neighborhood of $\sigma(T)$ (similar comment for Theorem 1). The condition $\left\|R_{\lambda}(T)\right\| \leqq K /(1-|\lambda|)$ for $|\lambda|<1$ is as general as condition (a). More generally, if $\left\|R_{\lambda}(T)\right\| \leqq K /$ dist $[\lambda, \sigma(T)]$ for $\lambda$ in a neighborhood of $\sigma(T)$, then $\left\|R_{\lambda}(T)\right\| \leqq K^{\prime} / \operatorname{dist}[\lambda, \sigma(T)]$ for all $\lambda$.

Since $\left\|R_{\lambda}(T)\right\| \leqq K /$ dist $[\lambda, \sigma(T)]$ if $T$ is similar to a normal operator, it is attractive to conjecture that Theorem 2 is still true under the weaker hypothesis $\left\|R_{\lambda}(T)\right\|$ $\leqq K /|1-| \lambda||$ for all $\lambda$. An example of A. S. Markus, which appears in the next section, reveals that such hopes are ill founded.

3. Operators similar to selfadjoint operators. In this section we will extend the results of $\S 2$ to the situation where the spectrum of $T$ lies on a straight line.

THEOREM 3. Let $\operatorname{Im} \sigma(T) \geqq 0$. If $\left\|R_{\lambda}(T)\right\| \leqq|\operatorname{Im} \lambda|^{-1}$ for $\alpha<\operatorname{Im} \lambda<0$, then $T$ is similar to a dissipative operator, that is, an operator with positive imaginary part. 
Proof. Let $A=(T-i)(T+i)^{-1}$; hence the spectrum of $A$ is contained in the unit disc. We now wish to show that $\left\|R_{\lambda}(A)\right\| \leqq(|\lambda|-1)^{-1}$ for $1<|\lambda|<\beta$. The relation between the resolvent of $T$ and that of $A$ is not as simple as the relation between the operators themselves. However, it is a routine calculation, along the lines of the proof of Lemma 1 , to show that if $\lambda=\xi+i \eta$ and $1+\xi^{2} \geqq-2 \eta$ then $\left\|(T-\lambda)^{-1}\right\| \leqq|\eta|^{-1}$ if and only if

$$
\left\|\left[A-\frac{\left(\xi^{2}-1\right)+2 i \xi}{1+\xi^{2}+2 \eta}\right]^{-1}\right\| \leqq \frac{1+\xi^{2}+2 \eta}{2|\eta|}
$$

The last inequality is just $\left\|R_{\mu}(A)\right\| \leqq(|\mu|-1)^{-1}$ in an outer neighborhood of $U$. Thus it follows from Theorem 1 that $A=Q C Q^{-1}$ where $C$ is a contraction. Since $T=i(1+A)(1-A)^{-1}$ we find that $Q T Q^{-1}=i(1+C)(1-C)^{-1}$. The last expression is a dissipative operator which completes the proof.

THEOREM 4. Let the spectrum of $T$ be real. Let

(a) $\left\|R_{\lambda}(T)\right\| \leqq K / \operatorname{Im} \lambda$ for $0<\operatorname{Im} \lambda<\alpha$, and

(b) $\left\|R_{\lambda}(T)\right\| \leqq|\operatorname{Im} \lambda|^{-1}$ for $\beta<\operatorname{Im} \lambda<0$.

Then $T$ is similar to a selfadjoint operator.

Proof. If we apply the last theorem to $T$ we find $T=Q A Q^{-1}$ where $A$ is dissipative. Clearly $\left\|R_{\lambda}(A)\right\| \leqq K^{\prime} / \operatorname{Im} \lambda$ for $0<\operatorname{Im} \lambda<\alpha$. It then follows immediately from [13, Theorem 7.1] that $A$ and hence $T$ is similar to a selfadjoint operator.

Alternatively, we may again set $A=(T-i)(T+i)^{-1}$. We have already seen that $A$ satisfies condition (b) of Theorem 2 . But it is easy to see that

$$
\begin{aligned}
\left\|(1-|\lambda|)(\lambda-A)^{-1}\right\| & \sim\left\|(1-\bar{\lambda} A)(\lambda-A)^{-1}\right\|=\left\|(\zeta-T)(\zeta-T)^{-1}\right\| \\
& \sim\left\|\operatorname{Im} \zeta(\zeta-T)^{-1}\right\|
\end{aligned}
$$

where $\zeta=i(1+\lambda) /(1-\lambda)$ and $|\lambda|<1$ hence $\operatorname{Im} \zeta>0$. Thus condition (a) is satisfied and the desired result follows from Theorem 2 .

REMARK. The growth condition(s) on $R_{\lambda}(T)$ in the last two theorems are really only needed in a neighborhood of the spectrum of $T$.

The condition that $\left\|R_{\lambda}(T)\right\| \leqq|\operatorname{Im} \lambda|^{-1}$ on one side of the real axis cannot easily be dispensed with in the last theorem. We will now sketch an example, due to A. S. Markus [18], of an operator $T$ where $\sigma(T)$ is real and $\left\|R_{\lambda}(T)\right\| \leqq K /|\operatorname{Im} \lambda|$ for all $\lambda$. However, $T$ is not similar to a selfadjoint operator.

Let $\left\{f_{n}\right\}$ be a Schauder basis for $\mathscr{H}$, which is not equivalent to an orthonormal basis (that is, there exists no orthonormal basis $\left\{\varphi_{n}\right\}$ such that $f_{n}=Q \varphi_{n}$ where $Q$ is an invertible operator). The existence of such a basis is extremely nontrivial. The first example, $\left\{\left(\alpha+\frac{1}{2}\right)|\chi|^{\alpha} e^{i n \pi x}\right\}_{-\infty}^{\infty}$ in $L^{2}[-1,1]$ where $0<|\alpha|<\frac{1}{2}$, is due to K. I. Babenko.

Let $\left\{\lambda_{n}\right\}$ be a decreasing sequence of real numbers, converging to 0 . Set $T f_{n}=\lambda_{n} f_{n}$, and extend $T$ to the rest of $\mathscr{H}$ by linearity. Then $T$ is a bounded transformation and $\left\|R_{\lambda}(T)\right\| \leqq K /|\operatorname{Im} \lambda|$ for all $\lambda$ (see [18] for details). However, $T$ cannot be similar 
to a selfadjoint operator, else $\left\{f_{n}\right\}$ would be equivalent to an orthonormal basis. In fact, $T$ cannot even be a spectral operator. If it were, it would follow from Theorem $\mathrm{D}$ of $\S 7$ that $T$ is scalar, which we have just seen is impossible. We note for future reference that the spectrum of $T$ consists precisely of the $\lambda_{n}$ 's, hence is countable.

It is also possible to construct an example with the desired properties from the work of McCarthy and Schwartz [20]. However, their example is less explicit.

4. Operators with countable spectra on smooth curves. Despite the fact that $\left\|R_{\lambda}(T)\right\| \leqq K /$ dist $[\lambda, \sigma(T)]$ does not imply that $T$ is spectral, as we have just seen, it is possible to weaken the conditions on $R_{\lambda}(T)$ if the spectrum of $T$ is sufficiently sparse.

Lemma 4. Let the spectrum of $T$ lie in $\Gamma$ where $\Gamma$ is a $C^{1}$ Jordan curve. Let

(a) $\left\|R_{\lambda}(T)\right\| \leqq\{\text { dist }[\lambda, \Gamma]\}^{-1}$ for $\lambda$ outside $\Gamma$,

(b) $\left\|R_{\lambda}(T)\right\| \leqq K /\{\text { dist }[\lambda, \Gamma]\}^{n}$ for $\lambda$ inside $\Gamma$, where $n$ is a positive integer. Let $\mu$ be an isolated point of $\sigma(T)$. Then

$$
M_{\mu}=\{f \in H: T f=\mu f\}
$$

is a nonempty, reducing subspace of $T$.

Proof. We may assume without loss of generality that $\mu=0$, and the tangent to $\Gamma$ at 0 is the $x$-axis. Let $\alpha, \beta$ be points on $\Gamma$ a distance $\zeta$ from 0 . (The points $\alpha, \beta$ are unique for $\zeta$ small.) Let $P=(2 \pi i)^{-1} \int_{\eta} R_{\lambda}(T) d \lambda$ when $\eta$ is a small circle containing 0 as the only point of $\sigma(T)$ in its interior. For $f \in P_{\mathscr{H}}$ we know that

$$
(T-\alpha)^{n}(T-\beta)^{n} f=\frac{1}{2 \pi i} \int_{\Gamma_{\zeta}}(\lambda-\alpha)^{n}(\lambda-\beta)^{n} R_{\lambda}(T) d \lambda
$$

where $\Gamma_{\zeta}$ is the quadrilateral cutting $\Gamma$ at $\alpha$ and $\beta$ and having transverse sides of length $2 \zeta$ (see Figure 1).

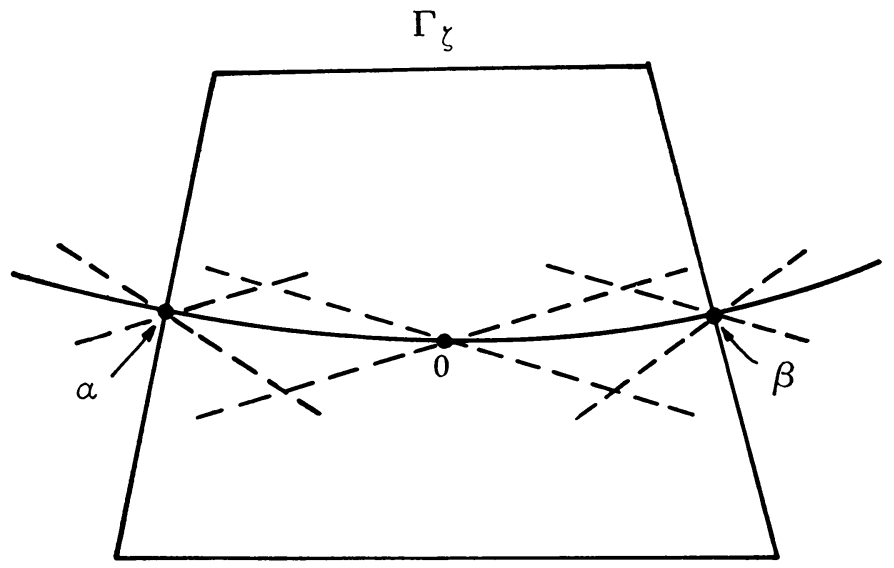

FIGURE 1 
It is not too difficult to see that

$$
\left\|(T-\alpha)^{n}(T-\beta)^{n} f\right\| \leqq M\left(|\zeta|+\varepsilon_{\zeta}\right)\|f\|
$$

where $M$ is independent of $\zeta$ and $\varepsilon_{\zeta} \rightarrow 0$ as $\zeta \rightarrow 0$. (A similar estimate is carried out in Bartle [1] when $\Gamma$ is a straight line.) To handle the general case we need the following observation. Let $\gamma: U \rightarrow \Gamma$ where $U$ is the unit circle. Let $\boldsymbol{n}(s)$ be the unit outer normal to $\Gamma$ at $\gamma(s)$. Define $f: U \times[-\delta, \delta] \rightarrow \boldsymbol{R}$ by

$$
\begin{array}{rlrl}
f(s, t) & =t^{-1} \operatorname{dist}[\gamma(s)+\operatorname{tn}(s), \Gamma] & \text { for } t & \neq 0, \\
& =1 & t & =0 .
\end{array}
$$

Then $f$ is continuous and, for $\delta$ sufficiently small, $f(s, t) \neq 0$. Thus there exists a constant $K$ such that

$$
\operatorname{dist}[\gamma(s)+\operatorname{tn}(s), \Gamma] \leqq K t \text { for }|t| \leqq \delta_{0} .
$$

If we let $\zeta \rightarrow 0$ we obtain $T^{2 n} f=0$ for $f \in P_{\mathscr{H}}$. Thus

$$
(\lambda-T)^{-1} f=\sum_{k=0}^{2 n-1} \lambda^{-(k+1)} T^{k} f
$$

for $f \in P_{\mathscr{H}}$ and $\lambda \notin \sigma(T)$. At this point we use the fact that

$$
\left\|(\lambda-T)^{-1}\right\| \leqq\{\operatorname{dist}[\lambda, \Gamma]\}^{-1}
$$

for $\lambda$ outside $\Gamma$, to conclude that $T f=0$ for $f \in P_{\mathscr{H}}$. It now follows from the corollary to Lemma 2 that $T^{*} f=0$ which completes the proof.

THEOREM 5. Let the spectrum of $T$ be countable and lie in the $C^{1}$ Jordan curve $\Gamma$. Let

(a) $\left\|R_{\lambda}(T)\right\| \leqq\{\text { dist }[\lambda, \Gamma]\}^{-1}$ for $\lambda$ outside $\Gamma$, and

(b) $\left\|R_{\lambda}(T)\right\| \leqq K /\{\text { dist }[\lambda, \Gamma]\}^{n}$ for $\lambda$ inside $\Gamma$.

Then $T$ is normal.

Proof. We begin by setting $T=T_{1} \oplus T_{2}$ on $\mathscr{H}_{1} \oplus \mathscr{H}_{2}$ where $T_{1}$ is the maximal reducing normal part of $T$ and $T_{2}$ is completely nonnormal. (It is easy to see that such a maximal part exists.) Since $T$ is the orthogonal direct sum of $T_{1}$ and $T_{2}$, it follows that $\sigma\left(T_{2}\right)$ is countable. Moreover, conditions (a) and (b) hold for $T_{2}$ in $\mathscr{H}_{2}$. We claim that $\mathscr{H}_{2}=\{0\}$ and thus $T$ is normal. Assume to the contrary that $\mathscr{H}_{2} \neq\{0\}$ and thus $\sigma\left(T_{2}\right) \neq \varnothing$. It follows from Lemma 4 and the maximality of $T_{1}$ that $\sigma\left(T_{2}\right)$ has no isolated points. But then $\sigma\left(T_{2}\right)$ must be a nonvoid, countable, perfect set which is impossible. The contradiction completes the proof.

COROLlaRY. Let $T$ be a contraction with $\sigma(T) \subset U$, and $\sigma(T)$ countable. If $\left\|R_{\lambda}(T)\right\|$ $\leqq K /(1-|\lambda|)^{n}$ for $|\lambda|<1$, then $T$ is unitary.

REMARK. The theorem obviously remains true if we interchange the words inside and outside in conditions (a) and (b). Moreover, we need these conditions only in a 
neighborhood of $\sigma(T)$. Since the example of A. S. Markus in the preceding section had countable spectrum, it is not possible to replace the 1 by $K$ in condition (a). Although the condition $\sigma(T)$ countable may appear unduly restrictive, the next section will reveal its pertinence.

Note that in Theorem 5, the spectral theoretic nature of $T$ is determined by the stronger growth condition, namely dist ${ }^{-1}$, and $T$ is normal. In Theorem 2, the spectral nature of $T$ is determined by the weaker condition $K /$ dist and $T$ is only similar to a normal operator.

5. An operator with some unusual properties. In this section we will construct an operator which places stringent limitations on any generalization of Theorems 2 and 5 . The operator will have very sparse spectrum and satisfy growth conditions on the resolvent, but will not be spectral. Before we can exhibit the operator we will need some results on inner functions which appear to be of interest in their own right. We begin with the following elementary lemma.

Lemma 5. Let $f$ be positice and decreasing on $[0,1]$. Let $\mu$ be continuous and increasing on $[0,1]$ with modulus of continuity $\omega(\cdot)$. Then for $\delta>0$,

$$
\int_{0}^{1} f(t) d \mu(t) \leqq \omega(\delta) f(0)+\frac{\omega(\delta)}{\delta} \int_{0}^{1} f(t) d t .
$$

Proof. Divide the unit interval into subintervals of length $\delta$ starting from the left (perhaps leaving an odd-sized interval). Then

$$
\int_{0}^{1} f(t) d \mu(t) \leqq \sum_{1}^{n} f\left(t_{i-1}\right)\left[\mu\left(t_{l}\right)-\mu\left(t_{i-1}\right)\right] .
$$

The lemma now follows by taking out the first term and renumbering the others to form a lower sum.

THEOREM 6. Let $\mu$ be an increasing continuous function on $[0,2 \pi]$. Set

$$
q(\lambda)=\exp \left(-\int_{0}^{2 \pi} \frac{e^{i t}+\lambda}{e^{i t}-\lambda} d \mu(t)\right)
$$

Then $|q(\lambda)| \geqq K(1-|\lambda|)^{\alpha}$ for $K, \alpha$ positive constants if and only if the modulus of continuity of $\mu$ is $O\left(t \log t^{-1}\right)$.

Proof. Assume that $\omega(t)$, the modulus of continuity of $\mu(t)$, is $O\left(t \log t^{-1}\right)$. Clearly

$$
|q(\lambda)|=\exp \left(-\int P_{r}(\theta-t) d \mu(t)\right)
$$

where $\lambda=r e^{i \theta}$ and $P_{r}$ is the Poisson kernel. It is sufficient to check the case $\lambda=r$, for the constants involved are independent of the argument. If we apply Lemma 5 
to both the top and bottom half of the circle with $f(t)=P_{r}(t)$ and $\delta=1-r$, we obtain

$$
\begin{aligned}
\int P_{r}(t) d \mu(t) & \leqq 2 K \log (1-r)^{-1}+K \log (1-r)^{-1} \int P_{r}(t) d t \\
& =K_{0} \log (1-r)^{-1}
\end{aligned}
$$

for $(1-r)$ sufficiently small. Thus $|q(r)| \geqq(1-r)^{K_{0}}$ for $r$ sufficiently close to 1 . Since $q(\lambda)$ never vanishes in the disc there exists an $M>0$ such that $|q(r)| \geqq$ $M(1-|\lambda|)^{K_{0}}$ for all $\lambda \in D$.

Conversely, let us assume the modulus of continuity is not $O\left(t \log t^{-1}\right)$. Then there exists a point, say 0 , such that $\mu(\delta)-\mu(0) \geqq K(\delta) \delta \log \delta^{-1}$ where $K(\delta) \rightarrow \infty$ as $\delta \rightarrow 0$. Hence

$$
\begin{aligned}
\int P_{r}(t) d \mu(t) & \geqq \int_{0}^{1-r} P_{r}(t) d \mu(t) \\
& \geqq P_{r}(1-r)[\mu(1-r)-\mu(0)] \geqq \frac{1}{2} K(1-r) \log (1-r)^{-1} .
\end{aligned}
$$

Thus it follows that $|q(r)| \leqq(1-r)^{K(1-r) / 2}$ which is a contradiction since $K(1-r)$ $\rightarrow \infty$ as $r \rightarrow 1$.

REMARK. If we divide $\mu$ by a constant, in effect we divide $\alpha$ by the same constant and thus we can ensure that $|q(\lambda)| \geqq K(1-|\lambda|)$ for suitably chosen $\mu$.

To construct our example we need to know that there exist singular measures (functions) with modulus of continuity $\delta \log \delta^{-1}$. This difficulty is taken care of by the next

THEOREM K (KAHANE [22]). There exists an increasing continuous singular function $\mu$ on $[0,2 \pi]$ with modulus of continuity $O\left(\delta \log \delta^{-1}\right)$ such that $\mu^{\prime}(t)$ exists and is 0 on an open set of measure $2 \pi$. Thus, the support of $\mu$, considered as a measure, is a nowhere dense, perfect set of measure 0 .

Actually Kahane proved more; his function is almost smooth (that is, $f(x+h)$ $+f(x-h)-2 f(x)=O(h))$. Since any almost smooth function has modulus of continuity $O\left(\delta \log \delta^{-1}\right)$ (see Zygmund [42, p. 44]), this is more than sufficient.

THEOREM 7. There exists an operator $T$ on the Hilbert space $H$ with the following properties:

(1) $T$ is a contraction.

(2) The spectrum of $T$ is a nowhere dense perfect set of measure zero on the unit circle.

(3) $\left\|(T-\lambda)^{-1}\right\| \leqq K /(1-|\lambda|)^{2}$ for $|\lambda|<1$.

(4) $T$ is not similar to a unitary operator, in fact $T$ is not even a spectral operator.

(5) $T \in C_{0}$ (in the sense of Sz.-Nagy-Foiaș; see the proof for definition), $T^{n} \rightarrow 0$ strongly and $T^{* n} \rightarrow 0$ strongly.

(6) Neither $T$ nor $T^{*}$ has any point spectrum.

(7) $T$ is a decomposable operator. 
Proof. Choose a singular function $\mu$ of the type described in the last theorem. Set

$$
q(\lambda)=\exp \left(-\int_{0}^{2 \pi} \frac{e^{2 t}+\lambda}{e^{2 t}-\lambda} d \mu(t)\right)
$$

We assume $\mu$ has been chosen so that $|q(\lambda)| \geqq K(1-|\lambda|)$ for $|\lambda|<1$. Let $H^{2}$ consist of those functions in $L^{2}[0,2 \pi]$ whose negative Fourier coefficients vanish. Set $H=H^{2} \ominus q\left(e^{i \theta}\right) H^{2}$. Let $P$ be the projection of $H^{2}$ on $H$. For $f \in H$ define $T f=P S f$ where $S f(\theta)=e^{i \theta} f(\theta)$ for $f(\theta) \in H^{2}$. (The operator $T$ just defined has been extensively studied for $q$ an arbitrary inner function and we refer the reader to [37, p. 244] and [32] for an excellent discussion.) We claim that $T$ has the properties listed above. Most can be read off rather easily from existing results. First it is well known and obvious that $T$ is a contraction. Second, the spectrum of $T$ consists precisely of the support of $\mu$ (see the proof of Theorems 6 and 7 of [32]). Next, since $q(\lambda)$ is the characteristic function of $T$ (usually written $\Theta_{T}(\lambda)$; see [33]), it follows from [13, p. 36] that

$$
\left|q(\lambda)^{-1}\right|=\left\|(1-\bar{\lambda} T)(\lambda-T)^{-1}\right\| \sim(1-|\lambda|)\left\|(\lambda-T)^{-1}\right\|
$$

and thus

$$
\left\|(\lambda-T)^{-1}\right\| \leqq K^{\prime} /(1-|\lambda|)^{2} .
$$

(A weaker, but adequate, inequality relating $q$ and $\left\|(\lambda-T)^{-1}\right\|$ can be found in [37, p. 334].) It is possible to prove that $T$ is not similar to a unitary in several ways. For example, it is implied by the second half of property (5). Also, it is not hard to see that $q$ takes on arbitrarily small values near singularities of $\mu$ and hence Theorem N-F implies $T$ cannot be similar to a unitary. To see that $T$ cannot be similar to a spectral operator, assume it is. Then it follows from Theorems D and 9 of $\S 7$ that $T$ is similar to a unitary which we know is impossible. Theorem 6 of [32] tells us that $\lim _{r \rightarrow 1} q(r T)=0$ in the strong topology, hence, $T \in C_{0}$ by definition. But if $T \in C_{0}$ then $T^{n} \rightarrow 0$ strongly and $T^{* n} \rightarrow 0$ strongly by Theorem 4 of [32]. Any $T \in C_{0}$ is decomposable by [11]. (An operator $T$ on $\mathscr{X}$ is decomposable if given $G_{i}$ open, $i=1, \ldots, n$, with $\sigma(T) \subset \bigcup G_{i}$ then there exist spectral maximal subspaces $L_{i}$, $i=1, \ldots, n$, such that $\mathscr{X}=L_{1}+\cdots+L_{n}$ and $\sigma\left(T \mid L_{i}\right) \subset G_{i}$.) Finally, since $T^{n}$ and $T^{* n}$ converge to 0 strongly, neither $T$ nor $T^{*}$ can have any point spectrum.

REMARK. The operator $T$ was constructed from the inner function $q$ which in turn was based on the measure $\mu$. There is another way to obtain a $q$ with the necessary properties. Let $\varphi$ be the mapping function from the unit disc to a nonSmirnov domain with boundary a rectifiable Jordan curve. Let $q$ be the inner part of $\varphi^{\prime}$ which is necessarily nontrivial. We claim that $q$ will also yield an operator satisfying all but property (2). The existence of such domains is due to Keldyš and Larentiev and later to Duren, Shapiro and Shields [8] with assistance from Piranian [22]. The verification of the relation $|q(\lambda)| \geqq K(1-|\lambda|)^{\alpha}$ for such functions can be 
found in Shapiro [26] (see Lemmas 1 and 2 in particular). C. Foiaș has observed that an operator satisfying (1) and (3) can be constructed by simply using the function $q(\lambda)=(1+\lambda) / 2$ as the characteristic operator function. The resulting operator would have the unit circle for its spectrum and would be quasi-similar to a unitary.

6. Operators with spectra lying on smooth curves. Theorem G-K can be rephrased in the following way: Let the unit disc $D$ be a spectral set for $T$ and let $\left\|R_{\lambda}(T)\right\| \leqq K /(1-|\lambda|)$ for $|\lambda|<1$. Then $T$ is similar to a unitary. In this section we wish to generalize the result to regions other than discs. (Gohberg and Krein have also treated the half plane case.)

Definition. A closed set $S$ is a spectral set for $T$ if $\|u(T)\| \leqq \sup _{\lambda \in S}\|u(\lambda)\|$ for all rational functions $u(\lambda)$ with poles off $S$.

THEOREM 8. Let $S$ be a compact, connected, simply connected set whose boundary $\Gamma$ is a $C^{2}$ Jordan curve. Let $S$ be a spectral set for $T$ and let $\left\|R_{\lambda}(T)\right\| \leqq K / \operatorname{dist}[\lambda, \Gamma]$ for $\lambda \in S$. Then $T$ is scalar, that is, similar to a normal operator.

Proof. Let $\varphi$ be the conformal map of Int $S$ to the open disc and let $f$ be the inverse map. Since $\Gamma$ is smooth, $f$ and $\varphi$ are homeomorphisms between $S$ and $D$. What is more, $f^{\prime}(t)$ exists, and is continuous and nonvanishing on $D$ by Kellogg's Theorem (see [38, p. 361] or [39]). Since $\varphi$ can be approximated uniformly on $S$ by polynomials, the operator $\varphi(T)$ is well defined and has $D$ as a spectral set (see [20]). Further, the spectrum of $\varphi(T)$ is contained in the unit circle $U$ by the spectral mapping theorem (see [10]). We now wish to check that the resolvent of $\varphi(T)$ satisfies a first order growth condition in the disc. Note first that for $\varphi(\mu)=\eta$ we have

$$
\begin{aligned}
\left\|(\varphi(T)-\varphi(\mu))^{-1}(T-\mu)\right\| & =\sup _{\lambda \in S}\left|\frac{\lambda-\mu}{\varphi(\lambda)-\varphi(\mu)}\right| \\
& =\sup _{|z|<1}\left|\frac{f(z)-f(\eta)}{z-\eta}\right| \leqq M
\end{aligned}
$$

where $M$ is independent of $\eta$ since $f^{\prime}$ is uniformly bounded in the disc. Thus

$$
\left\|(\varphi(T)-\eta)^{-1}\right\| \leqq M\left\|(T-\mu)^{-1}\right\| \leqq \frac{M_{1}}{\operatorname{dist}[\mu, \Gamma]} \leqq \frac{M_{2}}{\operatorname{dist}[\varphi(\mu), U]}=\frac{M_{2}}{1-|\eta|}
$$

since

$$
\operatorname{dist}[f(\eta), \Gamma] \geqq\left(f^{\prime}(\eta) / 4\right) \operatorname{dist}[\eta, U] \geqq(L / 4) \operatorname{dist}[\eta, U]
$$

by the Koebe-Bieberbach one quarter theorem, where $0<L=\inf _{|z|<1}\left|f^{\prime}(t)\right|$. We may now apply Theorem G-K to $\varphi(T)$ to conclude that $\varphi(T)=Q V Q^{-1}$ where $V$ is unitary. But then $T=f(\varphi(T))=Q f(V) Q^{-1}$ which completes the proof since $f(V)$ is normal. 
The restriction of connectivity, simple connectivity and compactness in the last theorem is unduly severe. If $S$ is not compact then we can reduce ourselves to the compact case by considering $(T-\mu)^{-1}$ instead of $T$ for a fixed $u \notin S$. If $S$ has a finite number of components then we just treat one component at a time. Since the "angle between the pieces" is bounded below, they can be straightened out by a similarity at the end. If $S$ is not simply connected we treat each component of the boundary separately, filling in the holes to obtain a super set for a spectral set. As above, gluing the pieces of the operator together affords no real difficulties. Thus we obtain the following

COROLlaRY. Let $S$ be a spectral set for $T$. Let $S$ have finitely many components where each component is finitely connected. Let the boundary $\Gamma$ of $S$ consist of disjoint $C^{2}$ Jordan curves. Let $\left\|R_{\lambda}(T)\right\| \leqq K /$ dist $[\lambda, \Gamma]$ for $\lambda \in S$. Then $T$ is similar to a normal operator.

We conclude this section with an application of Theorem 8 .

Proposition 1. Let $A$ and $B$ be compact, connected, simply connected sets with $C^{2}$ Jordan curves for boundaries. Let $A \cap B \subset \mathrm{Bdry} A$. Let $A$ be a spectral set for $T$ and $B$ a spectral set for $S$ where $T$ and $S$ are similar. Then $T$ is similar to a normal operator.

Proof. Since $B$ is a spectral set for $S$ it follows that $\left\|R_{\lambda}(S)\right\| \leqq\{\text { dist }[\lambda, B]\}^{-1}$ for $\lambda \notin B$. Hence

$$
\left\|R_{\lambda}(T)\right\|=K / \operatorname{dist}[\lambda, B] \leqq K / \operatorname{dist}[\lambda, \operatorname{Bdry} A]
$$

for $\lambda \in A$. It now follows from Theorem 8 that $T$ is similar to a normal.

Remark. C. Foiaș and J. William observed earlier that Proposition 1 is true when $A, B$ are the right and left half planes. If we strengthen the hypothesis of the proposition to make both $A$ and $B$ spectral sets for $T$ then it follows from Theorem 0 that $T$ is normal.

7. Growth conditions and spectral operators. We have already observed that an operator whose resolvent satisfies the condition $\left\|R_{\lambda}(T)\right\| \leqq K /$ dist $[\lambda, \sigma(T)]$ need not be spectral even if the spectrum of $T$ lies on a smooth curve. If, however, one knows at the outset that $T$ is spectral then the rate of growth of $R_{\lambda}(T)$ is closely connected with the behavior of $T$ (when $\sigma(T)$ is nice).

Definition. An operator $T$ on a Hilbert space is a spectral operator if $T=S+N$ where $S$ is similar to a normal, $N$ is quasi-nilpotent and $S$ and $N$ commute. (The operators $S$ and $N$ are uniquely determined. $S$ is called the scalar part.) We say that $T$ is a spectral operator of type $m-1$ if $N^{m}=0$.

This definition of spectral operator is not the one usually given, but it is equivalent for a Hilbert space and much more convenient for our purposes. (See [6].)

We first quote a theorem of Dunford which relates $R_{\lambda}(T)$ to the type of $T$ and then prove a mild generalization. 
THEOREM D. Let $T=S+N$ be a spectral operator on a Hilbert space where $\sigma(T)$ lies in a $C^{2}$ Jordan curve $\Gamma$. Let $\left\|R_{\lambda}(T)\right\| \leqq K /\{\text { dist }[\lambda, \Gamma]\}^{m}$ for all $|\lambda| \leqq\|T\|+1$. Then $T$ is of type $m-1$, that is, $N^{m}=0$.

(Theorem D is Theorem 18 of [7] or Theorem 3.21 of [5]. The theorem is also valid in a reflexive Banach space.)

THEOREM 9. Let $T=S+N$ be a spectral operator of type $m$. Let $\sigma(T)$ lie in a $C^{1}$ Jordan curve $\Gamma$. Let $\left\|R_{\lambda}(T)\right\| \leqq K /\{\text { dist }[\lambda, \Gamma]\}^{n}$ for $\lambda$ outside $\Gamma$, where $|\lambda| \leqq\|T\|+1$. Then $T$ is of type $n-1$, that is, $N^{n}=0$.

Proof. We assume $n<m+1$ since otherwise there is nothing to prove. We will first show that $N^{m}=0$. Since $T$ is of type $m$

$$
(\mu-T)^{-1}=\sum_{k=0}^{m} N^{k}(\mu-S)^{-(k+1)}
$$

for $\mu \notin \sigma(T)$. Thus if $\operatorname{dist}[\mu, \Gamma]=d$ then

$$
\left\|(\mu-T)^{-1} f\right\| \geqq\left\|(\mu-S)^{-(m+1)} N^{m} f\right\|-M d^{-m}\|f\|
$$

when $M$ is independent of $\mu$ and $d$ (for $d$ small). Since $S=Q A Q^{-1}$ where $A$ is normal, $(\mu-S)^{-(m+1)} N^{m} f=Q(\mu-A)^{-(m+1)} Q^{-1} N^{m} f$. Let $g=Q^{-1} N^{m} f$ and assume $g \neq 0$. Then

$$
\left\|(\mu-A)^{-(m+1)} g\right\|^{2}=\int_{\sigma(T)}|\mu-\lambda|^{-2(m+1)} d\|E(\lambda) g\|^{2} .
$$

Since the length of $\Gamma$ is finite and the total variation of $\|E(\lambda) g\|^{2}=\|g\|^{2}$, for every $\delta>0$ we can find a subarc $\Delta$ in $\Gamma$ of length $\delta$ such that the total variation of $\|E(\lambda) g\|^{2}$ over $\delta$ is at least $\delta\|g\|^{2} / l(\Gamma)$. Since $\Gamma$ is continuously differentiable, it follows that $|\mu-\lambda| \leqq 2 d$ for $\lambda \in \Delta \subset \Gamma$ and dist $[\mu, \Gamma]=d$ sufficiently small where $\Delta$ is a subarc of length $\delta$. Thus for appropriately chosen $\mu$ and $\Delta$ we have

$$
\int_{\sigma(T)}|\mu-\lambda|^{-2(m+1)} d\|E(\lambda) g\|^{2} \geqq \int_{\Delta}|\mu-\lambda|^{-2(m+1)} d\|E(\lambda) g\|^{2} \geqq M_{1} d^{-(2 m+1)}
$$

where $M_{1}$ is independent of $d$. Thus for suitably chosen $\mu$ outside $\Gamma$ we have

$$
K\|f\| d^{-n} \geqq\left\|(\mu-T)^{-1} f\right\| \geqq M_{2} d^{-(m+1 / 2)}-M d^{-m}\|f\| .
$$

If we let $d \rightarrow 0$ this leads to a contradiction since $n \leqq m$. Thus $0=g=N^{m} Q^{-1} f$ and hence $N^{m} f=0$ for all $f \in H$ or $N^{m}=0$. The argument can be repeated to show that $N^{n}=0$.

REMARK. Clearly it is immaterial whether the growth condition $\left\|R_{\lambda}(T)\right\| \leqq$ $K /\{\text { dist }[\lambda, \Gamma]\}^{n}$ is satisfied for $\lambda$ inside or outside $\Gamma$ and in fact we need it only on a dense set near $\Gamma$.

We close this section with an example. In [5, p. 609], the following question is raised. If $T=S+N$ is a spectral operator with $\sigma(T)$ nowhere dense and $\left\|R_{\lambda}(T)\right\|$ $\leqq K /\{\text { dist }[\lambda, \sigma(T)]\}^{m}$ for all $|\lambda| \leqq\|T\|+1$, must $T$ be spectral of type $m-1$, that is, 
must $N^{m}=0$ ? (No assumption is made about the underlying space.) The question is answered there, only in the case when $\sigma(T)$ lies on a $C^{2}$ Jordan curve (Theorem D of this section). Theorem 12 of [6] claims to answer the question affirmatively. We shall now present an example which shows that if $T=S+N$ is a spectral operator and even if

(i) $\sigma(T)$ is countable,

(ii) $T$ is of finite type (that is, $N^{k}=0$ for some $k$ ),

(iii) $\sigma(T)$ lies on a rectifiable Jordan curve,

(iv) the underlying space is a Hilbert space,

then one still cannot conclude that $\left\|R_{\lambda}(T)\right\| \leqq K /\{\text { dist }[\lambda, \sigma(T)]\}^{m}$ implies $N^{m}=0$. We will first construct an operator $T$ where $\sigma(T)$ lies in a rectifiable Jordan curve and $\left\|R_{\lambda}(T)\right\| \leqq K\{\text { dist }[\lambda, \Gamma]\}^{-m}$ for $|\lambda| \leqq\|T\|+1$. Let $\gamma_{k}=\left\{z:|z|=k^{-2}\right\}$ for $k=1,2, \ldots$ Our curve will consist of the $\gamma_{k}$ 's slightly bent and tied together to form a Jordan arc (see Figure 2). It is easy to see that $\Gamma$ is rectifiable.

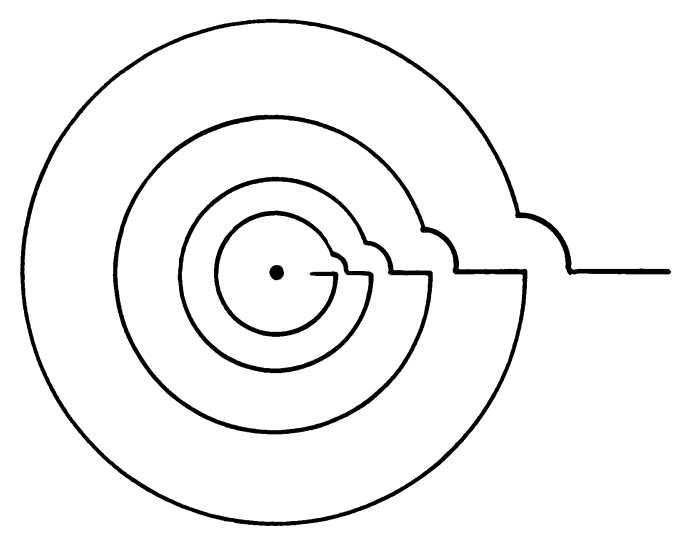

Figure 2

If $n^{-2} \leqq|z| \leqq(n-1)^{-2}$ then dist $[z, \Gamma] \leqq 2 n^{-3}$. Let $T_{1}$ be a nilpotent operator of order 3. Then $\left\|R_{\lambda}\left(T_{1}\right)\right\| \leqq M|\lambda|^{-3}$ for $|\lambda| \leqq\left\|T_{1}\right\|+1$. It is easy to see that $\left\|R_{\lambda}\left(T_{1}\right)\right\|$ $\leqq M_{1} /\{\operatorname{dist}[\lambda, \Gamma]\}^{2}$ for $|\lambda| \leqq\left\|T_{1}\right\|+1$. Thus we have constructed an operator satisfying our intermediate assertion. We define $T_{2}$ to be a diagonal (normal) operator with eigenvalues $\lambda_{j} \in \Gamma$ for $j=1,2, \ldots$ The $\lambda_{j}$ 's are so chosen that they accumulate only at zero and dist $\left[z,\left\{\lambda_{j}\right\}\right] \leqq 4 n^{-3}$ for $n^{-2} \leqq|z| \leqq(n-1)^{2}$. Having done this, we set $T=T_{1} \oplus T_{2}$. Clearly $T$ is a spectral operator of finite type. Further, $\left\|R_{\lambda}(T)\right\| \leqq M_{2}\{\text { dist }[\lambda, \sigma(T)]\}^{-2}$ for $|\lambda| \leqq\|T\|+1$. However, it is clear that $T_{1}$, the nilpotent part of $T$, does not satisfy the relation $T_{1}^{2}=0$. If one desires a Jordan curve instead of an arc, it is easy enough to connect into the origin.

More generally, the above technique will produce spectral operators of type $m-1$ whose resolvents will have growth of order $2 \mathrm{~m} / 3$. Thus the gap between the real type and the "resolvent type" of $T$ can be made arbitrarily large. Unfortunately 
the technique fails (for $m=2$ ) to produce a nonscalar $T$ whose resolvent has first order growth.

In view of the Markus example described earlier, the condition that $T$ be spectral to begin with is essential in the previous theorems. It is also necessary to restrict $T$ to be of finite type in Theorem 9. If $T$ is the Volterra operator $\left(f \rightarrow \int_{0}^{x} f(t) d t\right.$ for $\left.f \in L^{2}[0,1]\right)$, then $T$ is spectral; its spectrum lies on the $y$-axis and $\left\|R_{\lambda}(T)\right\| \leqq$ $|\operatorname{Re} \lambda|^{-1}$ for $\operatorname{Re} \lambda<0$. However $T$ is clearly not of type 0 , that is, scalar.

8. Additional examples. In this section we present several examples which did not arise in previous sections but which bear on the relation between an operator and its resolvent.

EXAMPLE 1. If $\sigma(Q)=\{0\}$ and $\left\|(1 \pm Q)^{-1}\right\|=1$, must $Q=0$ ? In view of Lemma 3 , this is equivalent to asking whether the condition $\left\|(t-Q)^{-1}\right\| \leqq(t)^{-1}$ for $-1 \leqq t \leqq 1$ implies $Q=0$. It is easy to see that $\left\|(t-Q)^{-1}\right\| \leqq|t|^{-1}$ for $-\infty<t<\infty$ does imply $Q=0$.

The answer to the original question, however, is no. The following example in modified form was suggested by $\mathrm{C}$. Foiaș. Let $T$ be a nonunitary contraction where $\sigma(T)=\{1\}$. (For example, let $T$ be the Cayley transform of the Volterra operator $V$ where $V: f(x) \rightarrow \int_{0}^{x} f(t) d t$ for $f \in L^{2}[0,1]$.) Let $S$ be the region in the complex plane bounded by the curves

$$
\begin{array}{ll}
z=i t, & 0 \leqq t \leqq 1, \\
z=t^{2}+i t, & 0 \leqq t \leqq 1, \\
z=t+i, & 0 \leqq t \leqq 1 .
\end{array}
$$

Let $\varphi$ be the conformal map of the unit disc onto $S$ where $\varphi(1)=0$. Let $Q=\varphi(T)$. Then $\left\|(Q \pm 1)^{-1}\right\|=1$. Since $\varphi^{-1}(Q)=\varphi^{-1}[\varphi(T)]=T \neq 0$, it follows that $Q \neq 0$.

This example can also be applied to a more interesting situation. If $\sigma(A) \subset[0,1]$ and $\left\|(A-\lambda)^{-1}\right\| \leqq|\operatorname{Im} \lambda|^{-1}$ for $0 \leqq \operatorname{Re} \lambda \leqq 1$ and $|\operatorname{Im} \lambda|<\beta$, must $A$ be selfadjoint ? (Put another way, must the growth condition in Theorem 0 hold in a neighborhood of $\sigma(T)$ or must it be just transversal to $\sigma(T)$ ?) By taking the direct sum of a selfadjoint operator and $i Q$, we can construct an example which answers this question in the negative. It should be observed that a stronger version of Theorem 0 (see [29]) implies such an $A$ is spectral.

EXAMPLE 2. It is easy to construct an operator $T$ where the numerical range of $T$ is contained in the unit disc but where $T$ is not a contraction. However, if one also insists that $\sigma(T) \subset U$, the unit circle, it becomes less obvious that such an example exists. (Equivalently, one could ask whether $\sigma(T) \subset U$ and $\left\|R_{\lambda}(T)\right\| \leqq(|\lambda|-1)^{-1}$ for $|\lambda| \geqq 1$ implies that $T$ is a contraction.) To construct such an example we shall first need a nonzero quasi-nilpotent operator $Q$ whose numerical range lies in a sector $S$ with vertex at the origin and with angular opening less than $\pi$. (For convenience we may assume $S$ is symmetric with respect to the real axis.) Such a $Q$ can be obtained as the square root of the Volterra operator (see [14, p. 281]) or by a method similar to the one employed in our first example. Having secured $Q$, we 
set $T=\alpha Q-1$ where $\alpha$ is to be specified. Let $W(T)^{-}$denote the closure of the numerical range of $T$. We choose $\alpha$ to be that unique positive real number such that $W(\alpha Q-1)^{-} \subset D$ and $W(\alpha Q-1)^{-} \cap U$ contains a point $\mu$ other than -1 . Since $W(T)$ is a continuous (upper semicontinuous set valued) function this is possible. We claim that $T=\alpha Q-1$ is not a contraction. For if $\mu \in W(T)^{-} \cap\{z:|z|=\|T\|\}$, then $\mu \in \sigma(T)$ and clearly $\mu \notin \sigma(\alpha Q-1)$.

The example we have just constructed can be used for another purpose. If we set $A=i(T+1)(T-1)^{-1}$ where $T$ is the above operator, we obtain an operator with the following properties:

(i) $\sigma(A)$ is real, in fact $\sigma(A)=\{0\}$.

(ii) $\left\|(A-\lambda)^{-1}\right\| \leqq(\operatorname{Im} \lambda)^{-1}$ for $0<\operatorname{Im} \lambda \leqq \frac{1}{2}$.

However it is not true that $\left\|(A-\lambda)^{-1}\right\| \leqq(\operatorname{Im} \lambda)^{-1}$ for $0<\operatorname{Im} \lambda<\infty$. For if the last statement were true then $A$ would be the negative of a dissipative operator and $T$, the inverse Cayley transform of $A$, would be a contraction which it is not.

9. Open questions. Before stating the first problem we present some background material. Consider the following conditions:

(1) $\|T\| \leqq 1$

(2) $|W(T)| \leqq 1$,

(3) $\left\|R_{\lambda}(T)\right\| \leqq(|\lambda|-1)^{-1}$ for $1<|\lambda|<\alpha$,

(4) $\left\|T^{n}\right\| \leqq M$ for $n=1,2, \ldots$,

(5) $\left\|R_{\lambda}(T)\right\| \leqq K /(|\lambda|-1)$ for $|\lambda|>1$.

As it stands, (3) does not imply $\sigma(T) \subset D$ but if we add that restriction, then $(1) \Rightarrow(2) \Rightarrow(3) \Rightarrow(4) \Rightarrow(5)$. The implications are all easy or well known and not reversible. In particular, (3) is equivalent to $T^{n}=\rho P_{\mathscr{H}} U^{n} \mid \mathscr{H}$ for $n=1,2, \ldots$, which clearly yields (4). Suppose we require that both $T$ and $T^{-1}$ satisfy conditions from the list. Can one then infer that $T$ is unitary, similar to a unitary, or nothing of the kind? Except in one case the answer is known and summarized below.

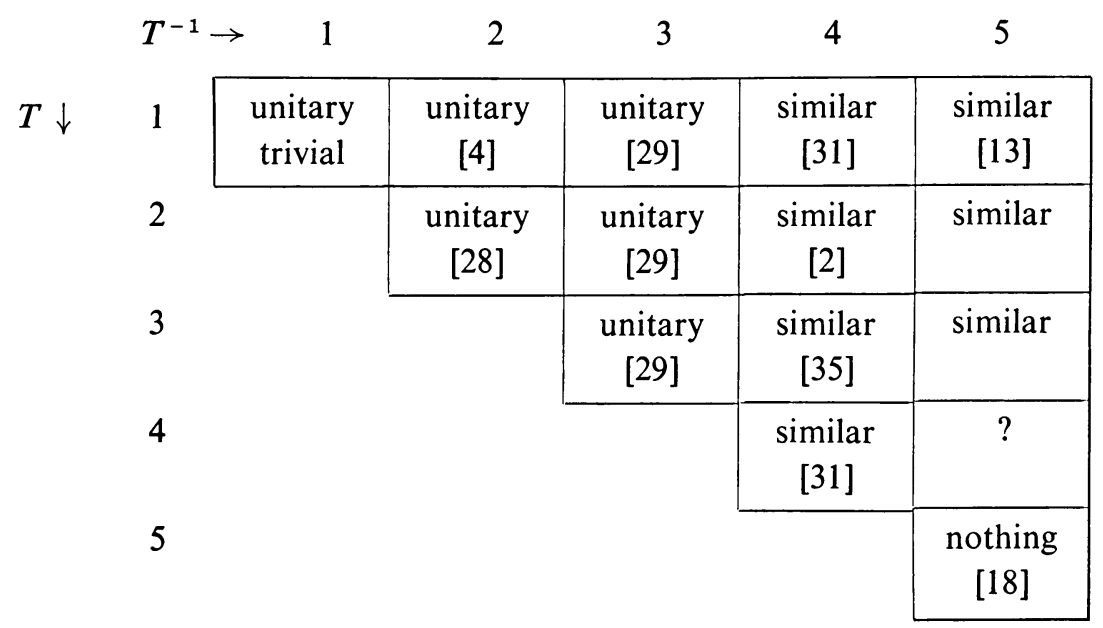


The reference to the paper which contains the result allows a wide range of immanence. If no reference is given, it can be found in the present paper. By slightly rephrasing the question in the box we arrive at

Question 1. If $\left\|T^{n}\right\| \leqq M$ for $n=1,2, \ldots$ and $\left\|R_{\lambda}(T)\right\| \leqq K /(1-|\lambda|)$ for $|\lambda|<1$ then must $T$ be similar to a unitary operator? The question can be modified by replacing " $\left\|T^{n}\right\| \leqq M$ for $n=1,2, \ldots$ ” by " $\|p(T)\| \leqq K\|p(\lambda)\|_{\infty}$ for all polynomials $p$ " where $\|p(\lambda)\|_{\infty}=\sup \{|p(\lambda)|=|\lambda| \leqq 1\}$.

Note that a negative solution would entail simultaneous construction of Foguel [9] and Markus [18] type examples.

The remaining problems arise naturally from the preceding sections.

Question 2. Let $\sigma(T)$ lie on a smooth Jordan curve $\Gamma$ and

(a) $\left\|R_{\lambda}(T)\right\| \leqq$ dist $[\lambda, \Gamma]^{-1}$ for $\lambda$ outside $\Gamma$,

(b) $\left\|_{\lambda}(T)\right\| \leqq K /$ dist $[\lambda, \Gamma]$ for $\lambda$ inside $\Gamma$.

Must $T$ be similar to a normal operator?

Hopefully an affirmative answer to Question 2 would contain Theorems 2, 4 and 8 as corollaries.

Question 3. Let $\sigma(T) \subset S$ where $S$ is a compact, connected, simply connected set with boundary a smooth Jordan curve $\Gamma$. If $\left\|R_{\lambda}(T)\right\| \leqq \operatorname{dist}[\lambda, S]^{-1}$ for $\lambda \notin S$ then is $T$ similar to an operator which has $S$ as a spectral set?

Note that an affirmative answer to 3 would yield an affirmative answer to 2 via Theorem 8 .

\section{REFERENCES}

1. R. G. Bartle, Spectral localization of operators in Banach spaces, Math. Ann. 153 (1964), 261-269. MR 29 \#1542.

2. C. A. Berger, A strange dilation theorem, Notices Amer. Math. Soc. 12 (1965), 590. Abstract \#625-152.

3. C. Davis and C. Foiaş, Operators with bounded characteristic function and their J-unitary dilation, Acta Sci. Math. (Szeged) 32 (1971), 127-139.

4. W. F. Donoghue, Jr., On a problem of Nieminen, Inst. Hautes Études Sci. Publ. Math. No. 16 (1963), 31-33. MR 27 \#2864.

5. N. Dunford, Spectral theory. II. Resolutions of the identity, Pacific J. Math. 2 (1952), 559-614. MR 14, 479.

6. - Spectral operators, Pacific J. Math. 4 (1954), 321-354. MR 16, 142.

7. - A survey of the theory of spectral operators, Bull. Amer. Math. Soc. 64 (1958), 217-274. MR 21 \#3616.

8. P. L. Duren, H. S. Shapiro and A. L. Shields, Singular measures and domains not of Smirnov type, Duke Math. J. 33 (1966), 247-254. MR 33 \#7506.

9. S. R. Foguel, A counterexample to a problem of Sz.-Nagy, Proc. Amer. Math. Soc. 15 (1964), 788-790. MR 29 \#2646.

10. C. Foiaş, Some applications of spectral sets. I: Harmonic-spectral measure, Acad. R. P. Romîne Stud. Cerc. Mat. 10 (1959), 365-401; English transl., Amer. Math. Soc. Transl. (2) 61 (1967), 25-62. MR 22 \#8340.

11. - The class $C_{0}$ in the theory of decomposable operators, Rev. Roumaine Math. Pures Appl. 14 (1969), 1433-1440. 
12. R. Godement, Théorème Taubériens et théorie spectrale, Ann. Sci. École Norm. Sup. (3) 64 (1947), 119-138. MR 9, 327.

13. I. C. Gohberg and M. G. Kreĭn, On a description of contraction operators similar to unitary ones, Funkcional. Anal. i Priložen. 1 (1967), 38-60= Functional Anal. Appl. 1 (1967), 33-52. MR 35 \#4761.

14. T. Kato, Perturbation theory for linear operators, Die Grundlehren der math. Wissenschaften, Band 132, Springer-Verlag, New York, 1966. MR 34 \#3324.

15. G. Leaf, A spectral theory for a class of linear operators, Pacific J. Math. 13 (1963), 141-155. MR 27 \#589.

16. E. R. Lorch, The integral representation of weakly almost-periodic transformations in reflexive vector spaces, Trans. Amer. Math. Soc. 49 (1941), 18-40. MR 2, 224.

17. V. I. Macaev, On a class of completely continuous operators, Dokl. Akad. Nauk SSSR 139 (1961), 548-551 = Soviet Math. Dokl. 2 (1961), 972-975. MR 24 \#A1617.

18. A. S. Markus, Some criteria for the completeness of a system of root vectors of a linear operator in a Banach space, Mat. Sb. 70 (112) (1966), 526-561; English transl., Amer. Math. Soc. Transl. (2) 85 (1969), 51-91. MR 35 \#7151.

19. C. A. McCarthy, The nilpotent part of a spectral operator, Pacific J. Math. 9 (1959), 1223-1231. MR 21 \#7442.

20. C. A. McCarthy and J. Schwartz, On the norm of a finite Boolean algebra of projections, and applications to theorems of Kreiss and Morton, Comm. Pure Appl. Math. 18 (1965), 191-201. MR 31 \#5097.

21. J. von Neumann, Eine Spektraltheorie für allgemeine Operatoren eines unitären Raumes, Math. Nachr. 4 (1951), 258-281. MR 13, 254.

22. G. Piranian, Two monotonic, singular, uniformly almost smooth functions, Duke Math. J. 33 (1966), 255-262. MR 33 \#7468.

23. C. R. Putnam, Eigenvalues and boundary spectra, Illinois J. Math. 12 (1968), 278-282. MR 37 \#2030.

24. L. A. Sahnovič, Nonunitary operators with absolutely continuous spectrum, Izv. Akad. Nauk SSSR Ser. Mat. 33 (1969), 52-64= Math. USSR Izv. 3 (1969), 51-64. MR 39 \#6107.

25. J. Schwartz, Subdiagonalization of operators in Hilbert space with compact imaginary part, Comm. Pure Appl. Math. 15 (1962), 159-172. MR 26 \#1759.

26. H. S. Shapiro, Remarks concerning domains of Smirnov type, Michigan Math. J. 13 (1966), 341-348. MR 34 \#4472.

27. J. G. Stampfli, Analytic extensions and spectral localization, J. Math. Mech. 16 (1966), 287-296. MR 33 \#4687.

28. —, Minimal range theorems for operators with thin spectra, Pacific J. Math. 23 (1967), 601-612. MR 37 \#4655.

29. — A local spectral theory for operators, J. Functional Analysis 4 (1969), 1-10. MR 39 \#4698.

30. — A local spectral theory for operators. II, Bull. Amer. Math. Soc. 75 (1969), 803-806. MR 39 \#6108.

31. B. Sz.-Nagy, On uniformly bounded linear transformations in Hilbert space, Acta Univ. Szeged. Sest. Sci. Math. 11 (1947), 152-157. MR 9, 191.

32. B. Sz.-Nagy and C. Foiaş, Sur les contractions de l'espace de Hilbert. VII. Triangulations canoniques. Fonction minimum, Acta Sci. Math. (Szeged) 25 (1964), 12-37. MR 30 \#455.

33. - Sur les contractions de l'espace de Hilbert. VIII. Fonctions caractéristiques. Modèles fonctionnels, Acta Sci. Math. (Szeged) 25 (1964), 38-71. MR 30 \#2348.

34. - Sur les contractions de l'espace de Hilbert. X. Contractions similaires à des transformations unitaires, Acta Sci. Math. (Szeged) 26 (1965), 79-91. MR 34 \#1856. 
35. B. Sz.-Nagy, On certain classes of power-bounded operators in Hilbert space, Acta Sci. Math. (Szeged) 27 (1966), 17-25. MR 33 \#6413.

36. - Similitude des opérateurs de classe $C_{p}$ à des contractions, C. R. Acad. Sci. Paris Sér. A-B 264 (1967), A1063-A1065. MR 35 \#3473.

37. —_ Analyse harmonique des opérateurs de l'espace de Hilbert, Masson, Paris; Akad. Kiadó, Budapest, 1967. MR 37 \#778.

38. M. Tsuji, Potential theory in modern function theory, Maruzen, Tokyo, 1959. MR 22 \#5712.

39. S. E. Warschawski, Über das Randverhalten der Ableitung der Abbildungsfunktion bei konformer Abbildung, Math. Z. 35 (1932), 321-456.

40. J. Wermer, The existence of invariant subspaces, Duke Math. J. 19 (1952), 615-622. MR 14, 384.

41. F. Wolf, Operators in Banach space which admit a generalized spectral decomposition, Nederl. Akad. Wetensch. Proc. Ser. A 60=Indag. Math. 19 (1957), 302-311. MR 19, 869.

42. A. Zygmund, Trigonometric series, 2nd ed., Cambridge Univ. Press, New York, 1968. MR 38 \#4882.

Department of Mathematics, Indiana University, Bloomington, Indiana 47401 